\title{
LncRNA UCA1 alleviates aberrant hippocampal neurogenesis through regulating miR-375/SFRP1-mediated WNT/ $\beta$-catenin pathway in kainic acid-induced epilepsy
}

 \\ Ling $\mathrm{Lu}^{3}$, Huan $\mathrm{Li}^{3}$ and Xianqiu $\mathrm{LiaO}^{3}$
}

1Department of Neurology, The First Affiliated Hospital of Guangxi University of Chinese Medicine, Nanning, Guangxi, 530023, China; 2Department of Numerical Control Technology, Guangxi Technological College of Machinery and Electricity, Nanning, Guangxi, 530007, China; ${ }^{3}$ Department of Graduate School, Guangxi University of Chinese Medicine, Nanning, Guangxi, 530023, China; ${ }^{4}$ Department of Laboratory, The First Affiliated Hospital of Guangxi University of Chinese Medicine, Nanning, Guangxi, 530023, China

\begin{abstract}
Temporal lobe epilepsy (TLE) is a chronic disease of the nervous system, associated with increased proliferation in the hippocampus. Urothcarcinoma associated 1 (UCA1) is a long long non-coding RNA that was shown to regulate proliferation and differentiation of neural progenitors in vitro. We hypothesised that TLE-associated abnormal proliferation is a consequence of the downregulation of UCA1. This hypothesis was tested in mice with kainic acid (KA)-induced seizures, and then the potential mechanism was explored in vitro and in vivo. Result showed that the expression of UCA1 and Secreted Frizzled Related Protein 1 (SFRP1) were significantly reduced in hippocampal tissues of epileptic mice, while miR-375 was increased compared with the control group. Pearson correlation analysis showed that UCA1 was positively correlated with SFRP1, while miR-375 was negatively correlated with UCA1 and SFRP1. Besides, UCA1 was overexpressed in mice and the overexpression of UCA1 significantly reversed the abnormal proliferation of hippocampal neurons in epilepsy mice. In vitro Luciferase assay showed that UCA1 and Sfrp1 are both the targets of miR-375, and UCA1 promotes the expression of Sfrp1 by competitively adsorbing miR-375, thereby inhibiting the activation of the WNT/ $\beta$-catenin pathway. The inactivation of the WNT/ $\beta$-catenin pathway prevented the abnormal proliferation of neural progenitors in the epileptic hippocampus. In conclusion, our findings provide a theoretical basis for the clinical application of UCA1.
\end{abstract}

Keywords: Temporal lobe epilepsy, Urothcarcinoma associated 1, miR-375, hippocampal neurogenesis, Secreted Frizzled Related Protein 1, WNT/ $\beta$-catenin pathway

Received: 11 August, 2020; revised: 13 October, 2020; accepted: 16 December, 2020; available on-line: 08 April, 2021

$\square$ e-mail: diaolimei0810@163.com

\#These authors contributed equally to the work.

Acknowledgements of Financial Support: This work was supported by the China National Natural Sciences Foundation (Grant No. 81960858), China National Natural Sciences Foundation (Grant No. 81760809) and Guangxi Natural Sciences Foundation (Grant no. 2017GXNSFAA198294).

Abbreviations: ANOVA, analyzed by one-way analysis of variance; KA, kainic acid; IncRNAs, long non-coding RNAs; miR-375 inh, miR375 inhibitor; miRNAs, microRNAs; MUT, mutant; NC inh, NC inhibitor; PFA, paraformaldehyde; SFRP1, Secreted Frizzled Related Protein 1; TLE, Temporal lobe epilepsy; UCA1, Urothcarcinoma associated $1 ; 3^{\prime}-U T R$, 3 untranslated-untranslated region; WT, wild type

\section{INTRODUCTION}

Temporal lobe epilepsy (TLE) is one of the most common chronic diseases of the nervous system, and its clinical treatment is extremely scarce worldwide (Bartolomei et al., 2008). It is well known that TLE is a very common locally related epilepsy, usually drug-resistant (Asadi-Pooya et al., 2017), and known as hippocampal sclerosis, which is characterized by the loss of a large number of neurons and severe glial hyperplasia (Tai et al., 2018). Abnormal neurogenesis and reorganization of neural circuits in the hippocampus cause spontaneous or recurrent seizures of epileptic foci and participate in the process of temporal lobe epilepsy (Cho et al., 2015). Although recent data shows that abnormal neurogenesis is caused by acute attacks or sudden injuries, the mechanism of abnormal neurogenesis in temporal lobe epilepsy is still elusive.

Studies have shown that long non-coding RNAs (long non-coding RNAs, lncRNAs) are also key regulators of the occurrence and development of diseases including epilepsy (Qiao et al., 2018). Urothcarcinoma associated 1 (UCA1) is a lncRNA originally found in bladder transitional cell carcinoma (Fan et al., 2014). Down-regulation of UCA1 plays a role in promoting apoptosis in primary cardiomyocytes by promoting p27 expression (Liu et al., 2015). Recently, UCA1 has been shown to promote the proliferation and differentiation of neural stem cells, suggesting that UCA1 may play an important role in the nervous system (Zheng et al., 2017). Recent studies have found that UCA1 has a certain role in epilepsy. For example, UCA1 inhibits epilepsy and epilepsy-induced brain injury by regulating miR-495/Nrf2-ARE signaling pathway (Zheng et al., 2017). UCA1 inhibits temporal lobe epilepsy star by regulating JAK/STAT signaling pathway Activation of shaped cells (Geng et al., 2018). However, the role and mechanism of UCA1 in abnormal neurogenesis caused by epilepsy has not been investigated.

Recently, the role of small non coding RNAs, especially microRNAs (miRNAs), in epilepsy and neurodegenerative diseases (Alzheimer's disease and Huntington's disease) has attracted great attention (Hébert et al., 2008; Johnson et al., 2008; Wu et al., 2019). MiR-375 has been reported to be involved in neuromodulation. For example, miR-375 inhibits neuronal differentiation by reducing HUD levels (Abdelmohsen et al., 2010), and miR-375 inhibits neuronal apoptosis and promotes growth in cerebral ischemia-reperfusion (Ou et al., 2017). It is reported that miR-375 is up-regulated in the KA- 
induced SD epilepsy rat model (Henshall 2013). However, whether the abnormal expression of miR-375 in the hippocampus of patients with temporal lobe epilepsy is related to neuronal hyperplasia has not been reported.

The current study explored the effect of UCA1 on hippocampal neurogenesis in KA-induced epilepsy mice and its potential molecular mechanism. It was found that UCA1 regulates the expression of Secreted Frizzled Related Protein 1 (Sfrp1) by targeting miR-375, thereby regulating the WNT/ $\beta$-catenin signaling pathway and participating in abnormal neurogenesis caused by epilepsy. Our findings provide a theoretical basis for the clinical application of UCA1.

\section{MATERIAL AND METHODS}

\section{Animal modeling and grouping}

All animal experiments were approved by the Guangxi University of Chinese Medicine Institutional Review Board. All procedures were conducted according to the Guide for the Use of Laboratory Animals (National Academy Press). 24 adult male C57Bl/6 mice (10 weeks, weighing 20-25g) were obtained from Hunan Slake Jingda Experimental Animal Co. LTD (SCXK(HU)2016-0002). The mice were housed in a constant environment (temperature $25^{\circ} \mathrm{C}$ ) with a $12-12 \mathrm{~h}$ light-dark cycle and free accesses of food and water. The mice were divided into two groups $(n=12)$ : Control group and kainic acid (KA) group. The modeling is carried out according to the method of Beamer et al., (Beamer et al., 2018). In short, the mice were deeply anesthetized with $5 \%$ isoflurane. Then, a guide sleeve was installed on the dura mater of mice and fixed with gutta percha for KA injection. KA was injected into the amygdala under a unilateral stereotaxic microscope $(0.3 \mu \mathrm{g}$ $\mathrm{KA}$ in $0.2 \mu$ l solvent, $\mathrm{pH}$ 7.4). Non epileptic control mice were injected with $2 \mu$ l of solvent in the same way. Three days later, the mice were exposed to Ad-vector, Ad-Uca1 or Ad-shSfrp1, respectively. Finally, the mice were euthanized by cervical dislocation, and PBS and 4\% paraformaldehyde (PFA) were perfused into the brain for analysis.

\section{Isolation of primary mouse hippocampal neurons}

After the hippocampal tissue was cut into pieces, Dhank's solution and $0.25 \%$ trypsin were added, gently blown and centrifuged, and all supernatant was collected. Sieve with 200 mesh and centrifuge for $5 \mathrm{~min}$ at $1500 \mathrm{r} /$ min. Discard supernatant, add medium to resuspend cells. Cells $\left(50000\right.$ cells $/ \mathrm{cm}^{2}$ ) were cultured in Neurobasal Media including 5\% FBS, B27, 2 mM GlutaMAX-I, penicillin/streptomycin and $15 \mathrm{mM}$ glucose at $37^{\circ} \mathrm{C}$ with $5 \% \mathrm{CO}_{2}$ in a humidified incubator, and transfected using Lipofectamine2000 (Invitrogen).

\section{UCA1 overexpression and SFRP1 knockdown}

UCA1 gene was integrated into pHBAd-U6 (Wujia, Beijing, China) to construct recombinant vector $\mathrm{pHBAd}-$ U6-UCA1. Ad-vector was used as a blank control. The successful construction of the vector was confirmed by sequencing and restriction enzyme digestion. The Sfrp1 shRNA-targeting sequence (sh\#1, 5'-CATGGCCTAACGGACGTAAA-3') or control shRNA sequence was inserted into pHBAd-U6 for adenovirus production. Mice in the normal group and KA group were injected with $1 \times 10^{7} \mathrm{pfu} / 10 \mu \mathrm{L}$ purified recombinant Ad-Ucal or
Ad-shSfrp1 intraperitoneally to overexpress UCA1 or knock down SFRP1.

\section{Cell culture}

HEK-293 cells were obtained from the Cell Bank of the Chinese Academy of Science (Shanghai, China) and maintained in DMEM (Thermo Scientific, San Diego, CA) supplemented with 10\% FBS (Thermo Scientific), penicillin $(100 \mathrm{U} / \mathrm{ml})$ (Thermo Scientific) and streptomycin $\left(100 \mu \mathrm{g} / \mathrm{ml}\right.$ ) (Thermo Scientific) at $37^{\circ} \mathrm{C}$ with $5 \% \mathrm{CO}_{2}$. All transfections were conducted using Lipofectamine2000 (Invitrogen).

\section{RT-qPCR}

The expression of Uca1, Sfrp1 and miR-375 in the hippocampus was detected. Total RNA was extracted from hippocampus or cells using Qiazol Lysis Reagent (Qiagen). RNAs were reverse transcribed to $\mathrm{cDNA}$ and quantified by qPCR using Quant One Step RT-qPCR Kit (SYBR Green) according to the manufacturer's protocol (Tiangen, Beijing, China). Relative quantities were calculated using $2^{\triangle \triangle C T}$ after normalization to Gapdh. Primers are as follows:

\section{Uca1, F, 5'-ACTCCGGACTAGCTGCAAGC-3', R, 5-TGC- CAACATACAACGTGGT-3';}

\section{Sfrp1, F, 5'-TAACTGCGGTAACT'TCCTTCAG-3', R, 5-CCT- TAAGCTGTTAACGTG-3';}

\author{
miR-375, F, 5'-CTGGACTATCGTAAACGT-3', R, \\ 5-TAACGTACGTAAACGTGCCG-3’;
}

\section{Brdu staining}

Three days after kainic acid administration and after shRNA administration, the mice were injected intraperitoneally with $\mathrm{BrdU}$ at $50 \mu \mathrm{g} / \mathrm{g}$ body weight 4 hours before sacrifice to mark the proliferating cells. BrdUpositive cells were detected using BrdU Labeling and Detection Kit I according to manufacturer's procedures (Roche, Indianapolis, IN)

\section{Dual luciferase reporter assay}

MiRDB (http://mirdb.org/) and TargetScan (http:// www.targetscan.org/vert_72/) were used to predict the targeted binding sites of miR-375 with Uca1 and Sfrp1, respectively. Subsequently, the hypothetical targeting relationship was further confirmed by dual luciferase reporter assay. In Brief, dual luciferase reporter gene vectors containing wild type (WT) sequence of Uca1 and Sfrp1 3 untranslated-untranslated region (3'-UTR) and mutant (MUT) sequence were constructed, which were named PmirGLO-Uca1-WT, PmirGLO-Uca1-MUT, PmirGLOSfrp1-WT and PmirGLO-Sfrp1-WT, respectively. Then, the recombinant plasmid and miR-375 mimic plasmid or NC plasmid or miR-375 inhibitor plasmid or NC inhibitor plasmid were co-transfected into HEK-293 cells respectively. After 24 hours, the cells were lysed and luciferase activity was detected by dual luciferase reporter gene detection system (E1910, Promega Corp., Madison, WI).

\section{RNA immunoprecipitation (RIP) assay}

RIP assay was conducted using magna RNA binding protein immunoprecipitation kit (Millipore) according to manufacturer's instructions. The cell lysate was incu- 
bated with human anti-Uca1 antibody (proteintech) or anti-mouse IgG coupled magnetic bead RIP buffer. Coprecipitated RNA was detected by qRT-PCR. To demonstrate that the detected RNA signals specifically bind to Uca1, we examined both total RNA (input control) and $\operatorname{IgG}$ controls.

\section{Western blotting}

Total protein in hippocampus or HEK-293 cells was lysed using Cell lysis buffer for Western and IP (Beyotime, Shanghai, China) according to the manufacturer's instructions. Cytoplasmic protein and nuclear protein were extracted by cytoplasmic protein extraction kit (BC3740, Solarbio, Beijing, China) and nuclear protein extraction kit (R0050, Solarbio, Beijing, China), respectively. Protein concentration was then measured using BCA Protein Assay Kit (Tiangen, Beijing, China), following protein separation by SDS-PAGE. Thereafter, the protein was then transferred to PVDF membrane, and then blocked with 5\% bovine serum albumin. After washed with TBST, the samples were incubated at $4^{\circ} \mathrm{C}$ overnight with primary antibodies to SFRP1 (1:230, ab4193, Abcam, Cambridge, UK), $\beta$-catenin (1:4000, ab6302, Abcam, Cambridge, UK) and $\beta$-actin (1:2000, ab8227, Abcam, Cambridge, UK). The next day, after washed with TBST for $10 \mathrm{~min}$, the samples were incubated with Goat Anti-Rabbit IgG H\&L (HRP) (ab205718, 1:30000, Abcam, Cambridge, $\mathrm{UK}$ ) at room temperature for $1 \mathrm{~h}$. Finally, the blots were analyzed using ImageJ 1.48u software (Bio-Rad, Hercules, CA, USA).

\section{Statistical analysis}

Data was exhibited as mean \pm standard derivation (mean \pm SD) and analyzed using SPSS 21.0 (IBM Corp. Armonk, NY, USA). Differences between two groups were analyzed by t-test, while differences between three groups or more were analyzed by one-way analysis of variance (ANOVA) following post hoc. Pearson was used to analyze the correlation between $U_{c a} 1$ and miR375, miR-375 and Sfrp1 and Uca1 and Sfrp1 expression. $p<0.05$ means significant difference.

\section{RESULTS}

\section{Correlation of Uca1, miR-375 and Sfrp1 expression in hippocampus of epileptic mice}

In this study, the expressions of Uca1, Sfrp1 and miR375 in hippocampal tissues of KA-induced epileptic mice were firstly investigated. As shown in Fig. 1A-C, the expression of Uca1 and Sfrp1 in hippocampal tissues of epileptic mice were significantly reduced, while the expression of miR-375 was significantly increased compared with the control group. Pearson correlation analysis showed that Uca1 was positively correlated with SFRP1, while miR-375 was negatively correlated with Uca1 and SFRP1 (Fig. 1D). These results indicated that abnormal expressions of Uca1, miR-375 and Sfrp1 were related to epilepsy.

\section{Uca1 overexpression reduced abnormal proliferation of neural progenitors in epileptic mice}

To investigate the effect of $U c a 1$ on the proliferation of neural progenitors in epileptic mice, Ucal was ectopically expressed. As shown in Fig. 2A, the expression of Uca1 in hippocampal nerve tissues of the Ad-Uca1 group was increased sharply in normal mice and epileptic mice compared with the Ad-vector group (Fig. 2A). Besides, BrdU staining showed that the overexpression of Ucal in normal mice had no significant effect on the prolif-
A

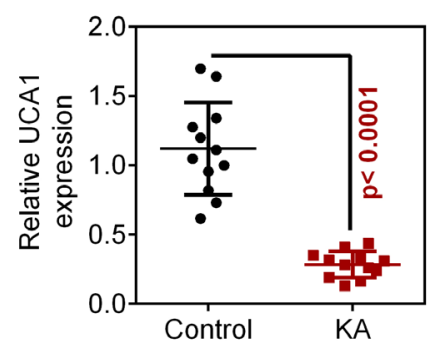

D

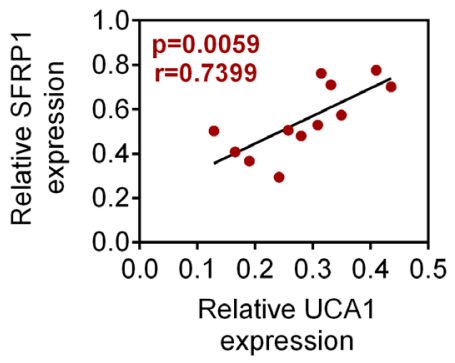

B
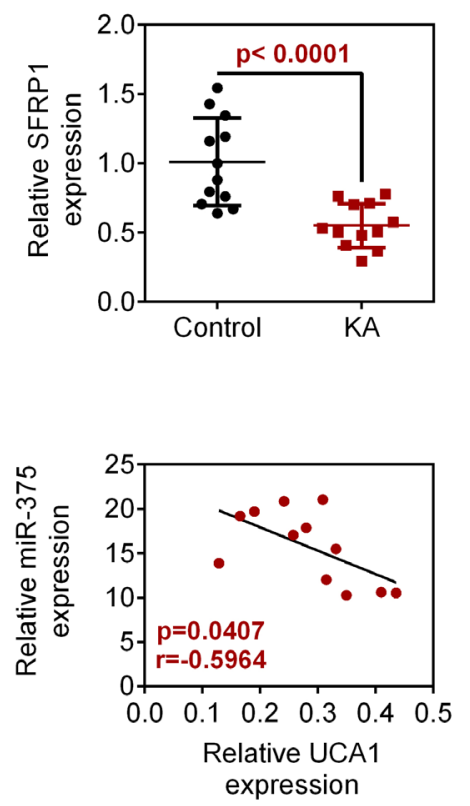

C
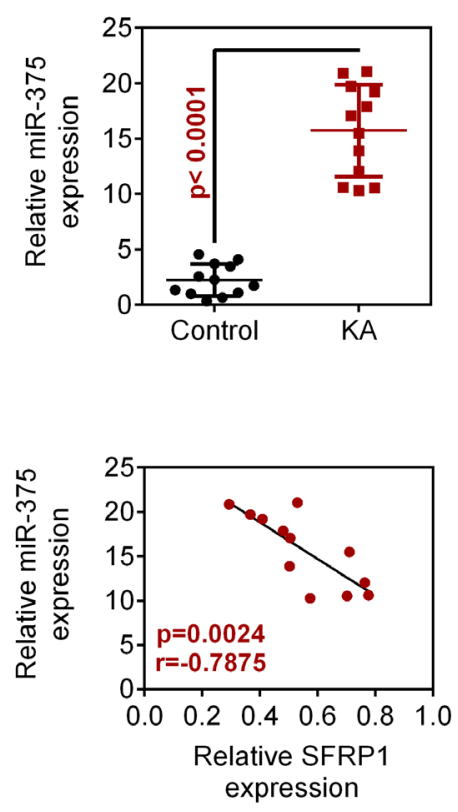

Figure 1. Correlation of Uca1, miR-375 and Sfrp1 expression in hippocampus of epileptic mice.

The mice were divided into two groups $(n=12)$ : Control group and KA group. A. The mRNA level of Uca1 was monitored by RT-qPCR. $(p<0.0001)$ B. The mRNA level of Sfrp 1 was monitored by RT-qPCR. $(p<0.0001) \mathrm{C}$. The mRNA level of miR-375 was monitored by RT-qPCR. $(p<0.0001)$ D. Pearson was used to analyze the correlation between Uca1 and miR-375, miR-375 and Sfrp1 and Uca1 and Sfrp1 expression. $(p<0.05)$ 
A



B

Control+Ad-vector



Control+Ad-UCA1
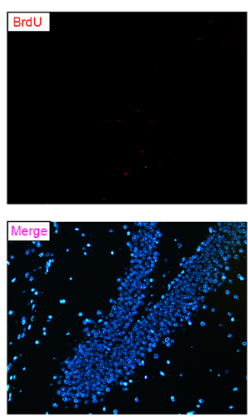
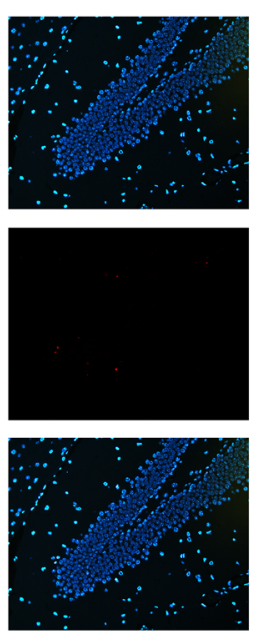

C

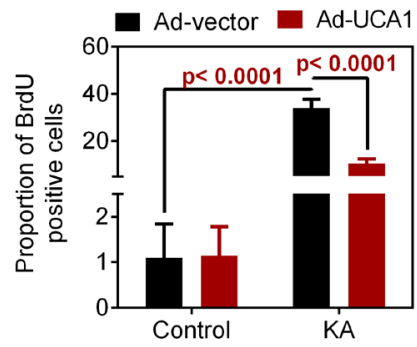

$\mathrm{KA}+\mathrm{Ad}$-vector

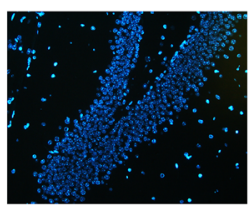

$\mathrm{KA}+\mathrm{Ad}-\mathrm{UCA}^{1}$
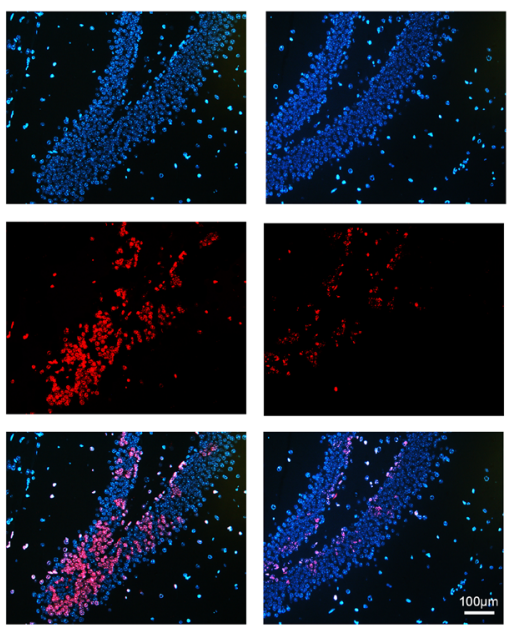

Figure 2. Up-regulation of Uca1 expression reduced abnormal proliferation of neural progenitors in epileptic mice.

The mice were divided into two groups $(n=12)$ : Control group and KA group. adenovirus Ad-vector or Ad-Ucal were infected into normal mice or epileptic mice. A. The mRNA level of Uca1 was monitored by RT-qPCR. $(p<0.0001)$ B-C. The proliferation of neural progenitors in hippocampus was monitored by Brdu staining. $(p<0.0001)$

eration of neural progenitors, but in epileptic mice, the number of BrdU positive neurons in Ad-vector group increased sharply. Surprisingly, overexpression of Uca1 significantly reduced the number of BrdU positive neurons in epileptic mice (Fig. 2B and C). In general, these results showed that up-regulation of Ucal expression reduced abnormal proliferation of neural progenitors in epileptic mice.

\section{Uca1 was combined with miR-375}

The target binding sites of miR-375 and Ucal was predicted by miRDB. (Fig. 3A). Besides, the targeting relationship between $\mathrm{miR}-375$ and Ucal was further confirmed by dual luciferase reporter assay and RIP. As shown in Fig. 3B, after co-transfection of Uca1-WT and miR-375 mimic into HEK-293 cells with Lipofectamine 3000 , the luciferase activity was decreased significantly, while after co-transfection of Uca1-WT and miR-375 inhibitor, the luciferase activity of HEK-293 cells was increased (Fig. 3B). RIP assay further showed that compared with $\operatorname{IgG}$, UCA1 was significantly enriched in Ago2, indicating that UCA1 directly binds to Ago2 (Figure 3C). qPCR further showed that $U_{c a 1}$ overexpression significantly reduced the expression of miR-375 in the hippocampal tissues of epileptic mice, but had no significant effect in normal mice (Fig. 3D). Together, these results suggest that Uca1 was combined with miR-375.
Uca1 overexpression promoted Sfrp1 expression through miR-375 to regulate WNT/ $\beta$-catenin pathway

The targeted binding site of miR-375 to Sfrp1 was predicted by TargetScan (Fig. 4A). Besides, the targeting relationship between miR-375 and Sfrp1 was further confirmed by dual luciferase report assay. As shown in Fig. 4B, after co-transfection of Sfrp1-W'T and miR-375 mimic into HEK-293 cells with Lipofectamine 3000, the luciferase activity of HEK-293 cells was decreased significantly, while after co-transfection of Sfrp1-W'T and miR375 inhibitor, the luciferase activity of HEK-293 cells was increased. Besides, after transfection of NC mimics, miR-375 mimic, NC inhibitor (NC inh) or miR-375 inhibitor (miR-375 inh) into neurons with Lipofectamine 3000, respectively, abnormal expression of Sfrp1 and $\beta$-catenin was observed. Western blotting further showed that overexpression of miR-375 significantly inhibited the expression of Sfrp1 in neurons and promoted the expression of $\beta$-catenin, while the low expression of miR375 showed the opposite effect (Fig. 4C). It is worth noting that Ucal overexpression significantly promoted the expression of Sfrp1, while suppressing the expression of $\beta$-catenin. Interestingly, miR-375 overexpression significantly reversed the effect of high expression of Uca1 on the expression of Sfrp 1 and $\beta$-catenin (Fig. 4D). Together, these results suggest that up-regulation of Ucal promoted Sfrp1 expression through miR-375 to regulate WNT/ $\beta$-catenin pathway. 
A

UCA1-WT 5 '...AAAATTTTTTCCACACCCAA AACAAA AA...3'

miR-375

UCA1-MUT

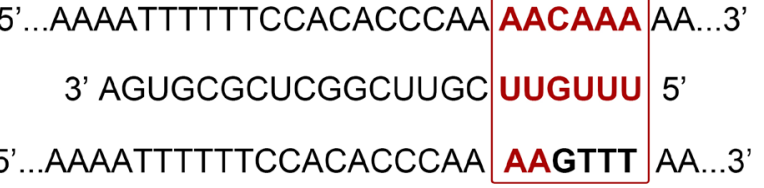

B
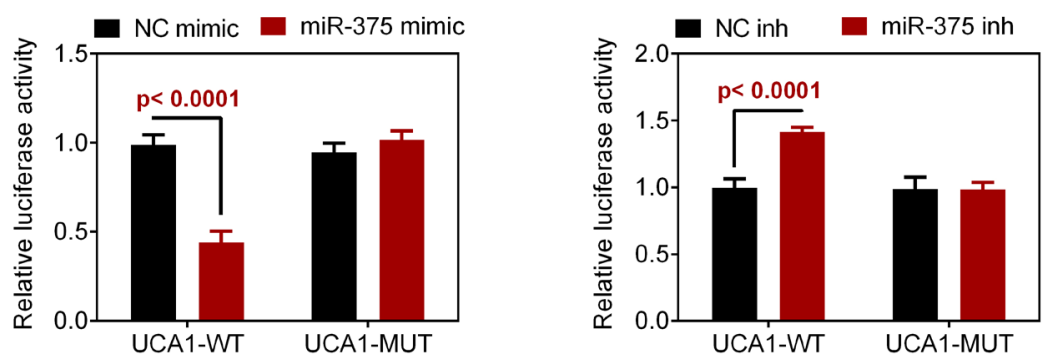

C

Anti-lgG $\square$ Input $\square$ Anti-Ago2
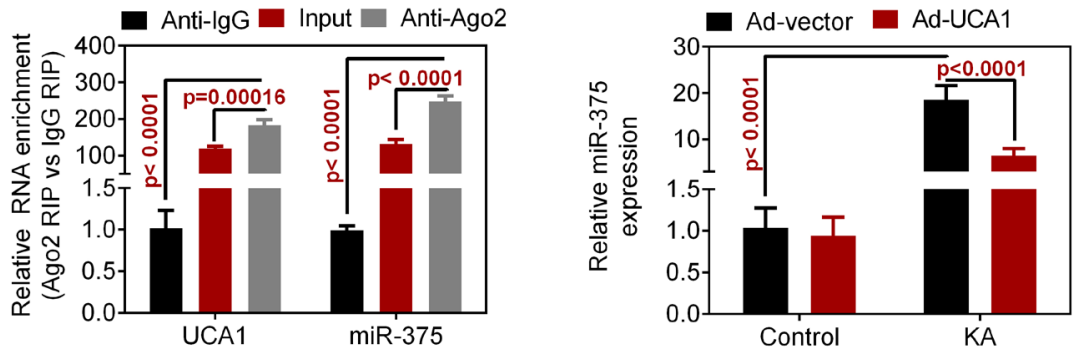

Figure 3. Uca1 was combined with miR-375.

A. The target binding sites of miR-375 and Uca1 was predicted by miRDB. B. Luciferase activity was monitored by dual luciferase reporter assay. $(p<0.0001) C$. The targeting relationship between miR-375 and Ucal was further confirmed by RIP assay. ( $p<0.0001) \mathrm{D}$. The mRNA level of miR-375 was monitored by RT-qPCR. $(p<0.0001)$

\section{Uca1 reduced the abnormal proliferation of neural progenitors in epileptic mice by regulating the expression of Sfrp1}

Finally, this study explored whether Sfrp1 is involved in the regulation of abnormal proliferation of neural progenitors by Uca1. Epileptic mice were injected with AdUca1 or/and shSfrp1 three days after kainic acid administration. The protein levels of SFRP1 and $\beta$-catenin was monitored by western blotting. As shown in Fig. 5A, Uca1 overexpression promoted the expression of Sfrp1 and suppressed the expression of $\beta$-catenin. After knockdown of Sfrp1 by shRNA in vivo, the expression of Sfrp1 was significantly reduced, while the expression of $\beta$-catenin was increased. Not only that, Sfrp1 knockdown reversed the effect of UCA1 on the expression of Sfrp1 and $\beta$-catenin. In addition, functional analysis showed that Uca1 overexpression inhibited the abnormal proliferation of neural progenitors, while sh-Sfrp1 showed the opposite effect, and Sfrp 1 knockdown reversed the inhibitory effect of Uca1 on abnormal neuronal proliferation (Fig. 5B). Taken together, these results indicate that Uca1 reduces the abnormal proliferation of neural progenitors in epileptic mice by regulating the expression of Sfrp1.

\section{DISCUSSION}

TLE is one of the most common types of intractable epilepsy, characterized by periodic seizures and unpredictability (Han et al., 2018). Long-term recurrent seizures or epileptic status in the developmental stage can cause many cognitive disorders, such as impairment of learning, memory, language, etc (Jones-Gotman et al., 1997; Boling 2018; Buck \& Sidhu 2020). Studies have shown that epileptic seizures are closely related to the abnormalities of hippocampal structure and function (Perkins et al., 2017). Epilepsy can cause the abnormal enhancement of hippocampal neurogenesis which is closely related to cognitive function (Cho et al., 2015). Therefore, it is very important to study the effect of epilepsy on hippocampal neurogenesis and early intervention. In this study, we successfully constructed kainic acid-induced epileptic mice model, and explored the role and potential molecular mechanism of lncRNA $U_{c a} 1$ in epileptic mice. The results found a new molecular mechanism of Uca1 in epilepsy, that is, the overexpression of Uca1 could significantly inhibit the abnormal proliferation of hippocampal neurons by WNT/ $\beta$-catenin pathway via regulating Sfrp1 expression. Our findings provided a basis for early intervention of epilepsy.

Epilepsy is closely related to the abnormal regulation of lncRNAs (Qiao et al., 2018; Villa et al., 2019). It was found that 384 or 279 lncRNAs were significantly deregulated in pilocarpine or KA-induced epilepsy mouse models (Lee et al., 2015). Uca1 is an oncogene and plays an important role in the development of tumors. Notably, Uca1 has been proved to promote the proliferation and differentiation of neural stem cells (Liu et al., 2015). In epilepsy, Geng et al., found that Ucal inhibited the apoptosis of hippocampal neurons, thus inhibiting the brain injury caused by epilepsy (Geng et al., 2018). The study of Wang et al revealed that Uca1 inhibited 
A
SFRP1-WT
5'... AAAACACATAGTTAAAAAAGA AACAAA TGA ...3'
miR-375
3' AGUGCGCUCGgCUUGC UUGUUU 5'
SFRP1-MUT
5'....AAAACACATAGTTAAAAAAGA tTGTAA TGA...3'

B
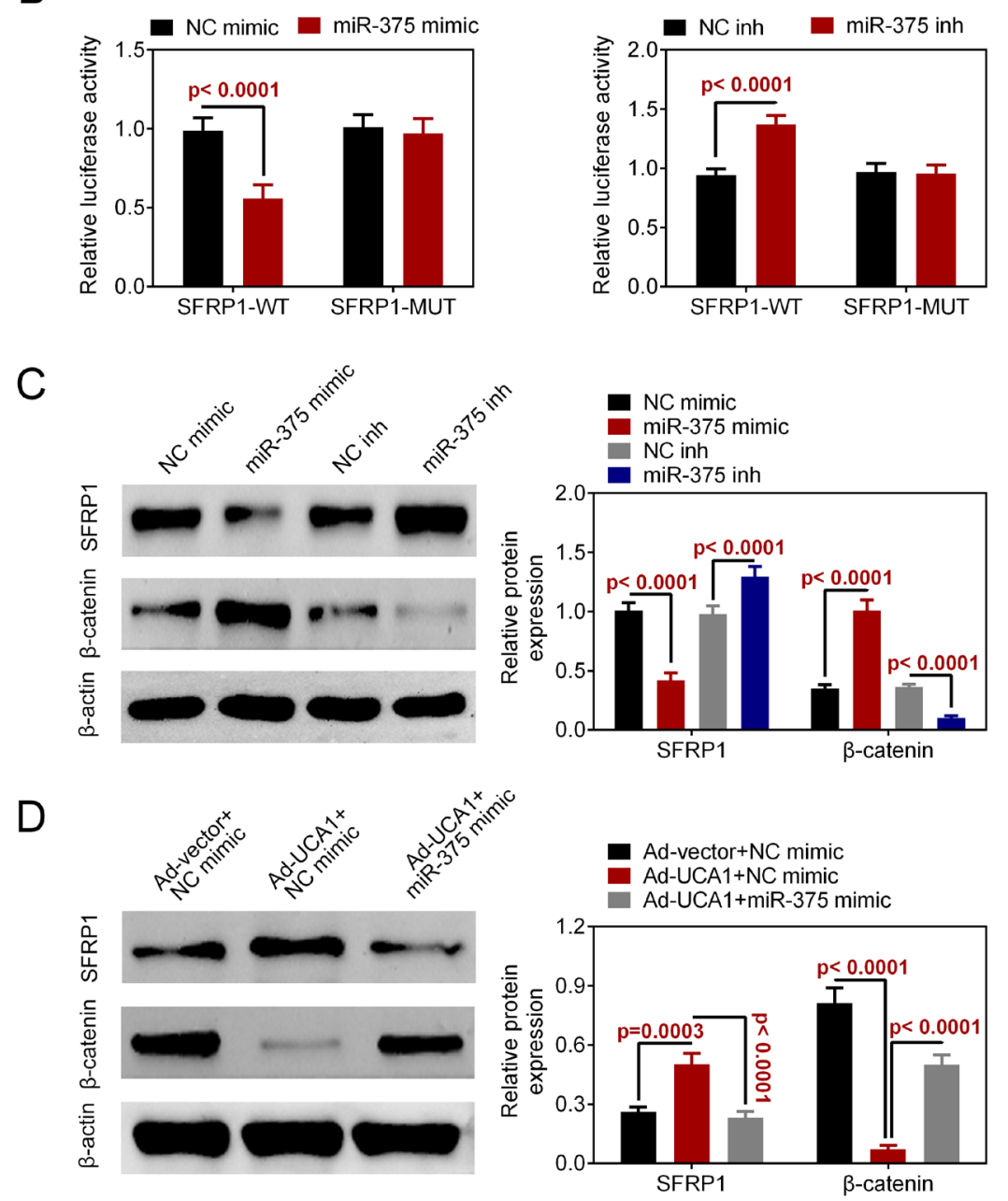

Figure 4. Up-regulation of Uca1 promoted Sfrp1 expression through miR-375 to regulate WNT/ $\beta$-catenin pathway.

The target binding sites of miR-375 and Sfrp 1 was predicted by TargetScan. B. Luciferase activity was monitored by dual luciferase reporter assay. $(p<0.0001)$ C. Neurons were transfected with NC mimic, miR-375 mimic, NC inhibitor or miR-375 inhibitor with Lipofectamine 3000 (Invitrogen), respectively. The protein levels of SFRP1 and $\beta$-catenin was monitored by western blotting. D. Neurons were co-transfected with Ad-vector and NC mimic or co-transfected with Ad-Ucal and NC mimic or miR-375 mimic. $(p<0.001)$

the activation of hippocampal astrocytes and improved the learning and memory ability of epilepsy rats, and had a protective effect on neuronal damage caused by KA. Further study clarifies that the role of Uca1 in epilepsy rats may be achieved by regulating the JAK/STAT signaling pathway (Wang et al., 2020). In addition, Yu et al., found that the expression of $U_{c a}$ in the SD rat epilepsy model established by lithium chloride and pilocarpine was also down-regulated. Uca1 overexpression can inhibit the epileptic inflammation by regulating the miR203/MEF2C/NF- $x \mathrm{~B}$ axis (Yu et al., 2020). Consistent with the above results, this study constructed a KAinduced mouse epilepsy model and found that $U_{c a} 1$ is low-expressed in epileptic mice. And the low expression of Uca1 caused abnormal proliferation of hippocampal neurons in epileptic mice. After forced overexpres- sion of $U c a 1$, the abnormal proliferation of hippocampal neurons was suppressed. Further mechanistic analysis showed that $U_{c a} 1$ could be targeted to downstream miR375 to promote the expression of Sfrp1, thereby inhibiting the activation of WNT/ $\beta$-catenin signaling pathway. Overall, this study considers Ucal as a potential target for clinical treatment of epilepsy.

There is increasing evidence that miRNAs exhibit abnormal regulation in epilepsy (Jimenez-Mateos et al., 2011; Hu et al., 2012). These abnormally regulated miRNAs mainly participate in the occurrence of epilepsy by regulating cell proliferation and migration, neuroinflammation and neuronal apoptosis (Karnati et al., 2015). MiR-375 is a widely studied miRNA that has been proved to participate in tumorigenesis (Wang et al., 2016; Kang et al., 2018). Studies have shown that 
A
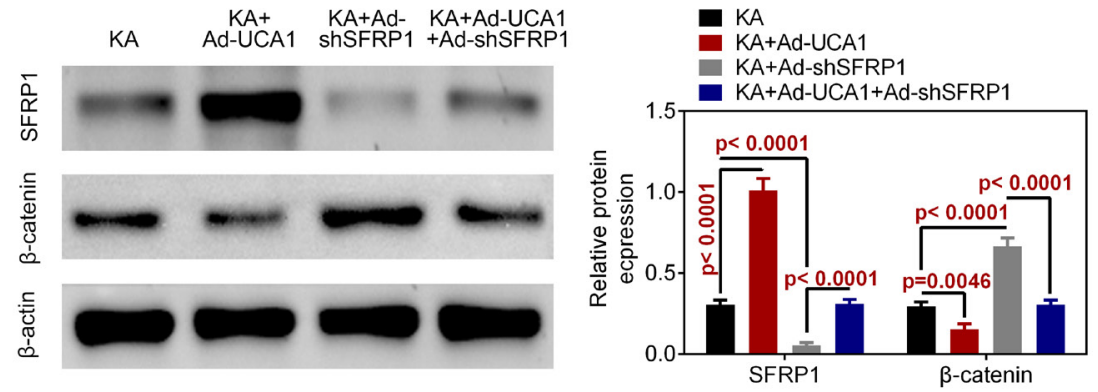

B

KA
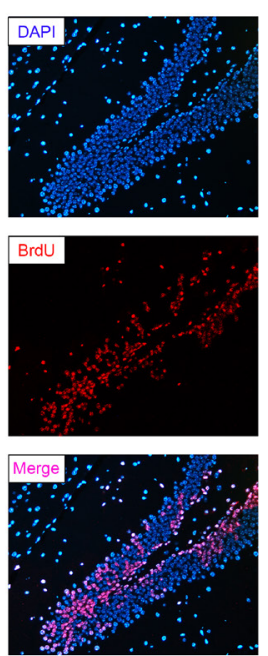

KA+Ad-UCA1
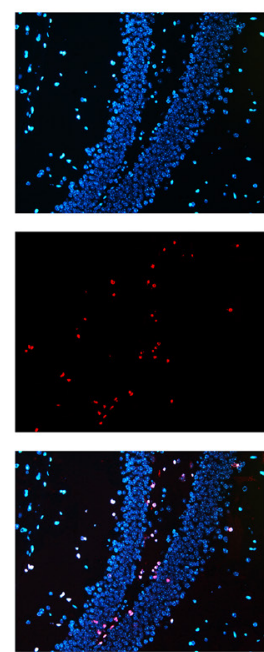

KA+Ad-shSFRP1
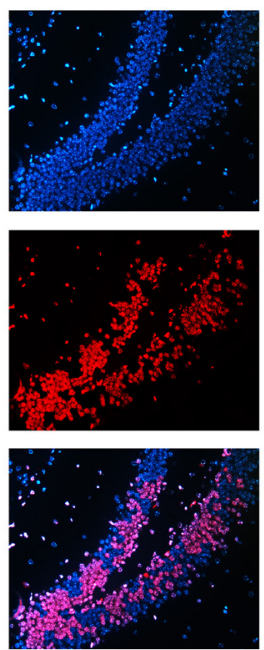

KA+Ad-UCA1+Ad-shSFRP1
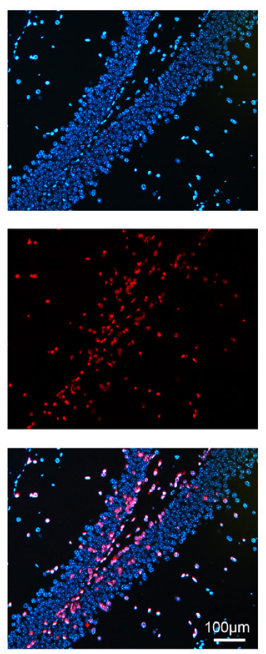

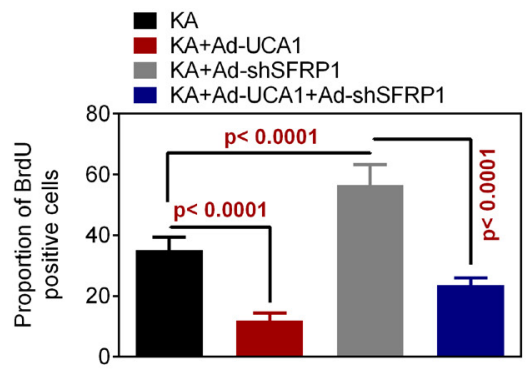

Figure 5. Up-regulation of Uca1 reduced nerve cell abnormal proliferation through SFRP1/WNT/ $\beta$-catenin pathway.

Epileptic mice were injected with Ad-Uca1 or/and shSfrp1 three days after kainic acid administration. A. The protein levels of SFRP1 and $\beta$-catenin was monitored by western blotting. $(p<0.0001) B$. The proliferation of neural progenitors in hippocampus was monitored by Brdu staining. $(p<0.0001)$

the expression of miR-375 was upregulated in the KAinduced SD epilepsy rat model (Henshall 2013), indicating that the abnormal expression of miR-375 may be related to the occurrence of epilepsy. However, the underlying molecular mechanism of the high expression of miR-375 for epilepsy remains unknown. In this study, a KA-induced epilepsy rat model was constructed, and the expression of miR-375 in the hippocampus tissues of epilepsy mice and normal mice was detected. Consistent with the above results, this study also found that miR-375 was highly expressed in KA-induced epilepsy mice. Further analysis showed that Uca1 targeted miR-375 and reduced the level of miR-375 in the hippocampus of epilepsy mice, which further inhibited the abnormal proliferation of hippocampal neurone. TargetScan analysis showed that Sfrp 1 was also a target of miR-375. Correlation analysis showed that miR-375 was negatively correlated with the expression of Uca1 and Sfrp1.

SFRP1 is a negative regulator of WNT signaling and dose-dependently regulate the development of midbrain dopamine neurons (Kele et al., 2012). SFRP1 participates in the regulation of $\mathrm{WNT} / \beta$-catenin pathway by suppressing the accumulation of $\beta$-catenin through a GSK-3 dependent mechanism, which interferes with the binding receptor of WNT and FRIZZLED protein (Kawano \& Kypta 2003; Galli et al., 2006). The WNT/ $\beta$-catenin pathway regulates hippocampal neurogenesis, synaptic division, and mitochondrial regulation, and is critical to the development and function of the central nervous system (Rubio et al., 2020). WNT/ $\beta$-catenin signals modulate epileptic neurogenesis and neuronal death. It also plays a role in the susceptibility of epilepsy and the development of chronic epilepsy, and has been found to be a 
promising antiepileptic target for the treatment of epilepsy in the future (Hodges \& Lugo 2018). In this study, it was found that miR-375 could promote the activation of WNT/ $\beta$-catenin pathway by targeting Sfrp1, thus promoting the abnormal proliferation of hippocampal neurone in epileptic mice. It is worth noting that UCA1 overexpression can inhibit the activation of $\mathrm{WNT} / \beta$ catenin pathway and prevent epilepsy by reducing the level of miR-375 and alleviating the inhibitory effect of miR-375 on Sfrp1.

In conclusion, we found that Uca1 was highly expressed in epileptic mice and miR-375 was poorly expressed. Further studies have shown that Uca1 can promote the expression of Sfrp 1 by reducing the level of miR-375, which further inhibits the abnormal proliferation of neural progenitors in epileptic mice by inhibiting the activation of the WNT/ $\beta$-catenin pathway.

\section{Acknowledgements}

Not applicable.

\section{Competing interests}

The authors state that there are no conflicts of interest to disclose.

\section{Ethics approval}

Ethical approval was obtained from the Guangxi University of Chinese Medicine Institutional Review Board.

\section{Statement of Informed Consent}

Written informed consent was obtained from a legally authorized representative(s) for anonymized patient information to be published in this article.

\section{Availability of data and materials}

All data generated or analyzed during this study are included in this published article.

\section{Authors' contributions}

Limei Diao, Haichun $\mathrm{Yu}$ and Huaqiong Li designed the study, supervised the data collection, Yueqiang $\mathrm{Hu}$, Mingfen $\mathrm{Li}$ and Qianchao $\mathrm{He}$ analyzed the data, interpreted the data, Ling Lu, Huan Li and Xianqiu Liao prepare the manuscript for publication and reviewed the draft of the manuscript. All authors have read and approved the manuscript.

\section{REFERENCE}

Abdelmohsen K, Hutchison ER, Lee EK, Kuwano Y, Kim MM, Masuda K, Srikantan S, Subaran SS, Marasa BS, Mattson MP, Gorospe M (2010) miR-375 inhibits differentiation of neurites by lowering HuD levels. Mol Cell Biol 30: 4197-4210. https://doi.org/10.1128/ mcb.00316-10

Asadi-Pooya AA, Stewart GR, Abrams DJ, Sharan A (2017) Prevalence and Incidence of Drug-Resistant Mesial Temporal Lobe Epilepsy in the United States. World Neurosurg. 99: 662-666. https://doi. org/10.1016/j.wneu.2016.12.074

Bartolomei F, Chauvel P, Wendling F (2008) Epileptogenicity of brain structures in human temporal lobe epilepsy: a quantified study from intracerebral EEG. Brain 131: 1818-1830. https://doi.org/10.1093/ brain/awn111

Beamer EH, Jurado-Arjona J, Jimenez-Mateos EM, Morgan J, Reschke CR, Kenny A, de Leo G, Olivos-Oré LA, Arribas-Blázquez M, Madden SF, Merchán-Rubira J, Delanty N, Farrell MA, O’Brien DF, Avila J, Diaz-Hernandez M, Miras-Portugal MT, Artalejo AR, Hernandez F, Henshall DC, Engel T (2018) MicroRNA-22 controls aberrant neurogenesis and changes in neuronal morphology after status epilepticus. Frontiers Mol Neurosci 11: 442-442. https://doi. org/10.3389/fnmol.2018.00442
Boling WW (2018) Surgical considerations of intractable mesial temporal lobe epilepsy. Brain Sci 8: 35. https://doi.org/10.3390/brainsci8020035

Buck S, Sidhu MK (2020) A guide to designing a memory fMRI paradigm for pre-surgical evaluation in temporal lobe epilepsy. Frontiers Neurol 10: 1354-1354. https://doi.org/10.3389/fneur.2019.01354

Cho KO, Lybrand ZR, Ito N, Brulet R, Tafacory F, Zhang L, Good L, Ure K, Kernie SG, Birnbaum SG, Scharfman HE, Eisch AJ, Hsieh J (2015) Aberrant hippocampal neurogenesis contributes to epilepsy and associated cognitive decline. Nat Commun 6: 6606-6606. https://doi.org/10.1038/ncomms7606

Cho KO, Lybrand ZR, Ito N, Brulet R, Tafacory F, Zhang L, Good L, Ure K, Kernie SG, Birnbaum SG, Scharfman HE, Eisch AJ, Hsieh J (2015) Aberrant hippocampal neurogenesis contributes to epilepsy and associated cognitive decline. Nat Commun 6: 6606. https://doi. org/10.1038/ncomms 7606

Fan Y, Shen B, Tan M, Mu X, Qin Y, Zhang F, Liu Y (2014) Long non-coding RNA UCA1 increases chemoresistance of bladder cancer cells by regulating Wnt signaling. FEBS J 281: 1750-1758. https://doi.org/10.1111/febs.12737

Galli LM, Barnes T, Cheng T, Acosta L, Anglade A, Willert K, Nusse R, Burrus LW (2006) Differential inhibition of Wnt-3a by Sfrp-1, Sfrp-2, and Sfrp-3. Dev Dyn 235: 681-690. https://doi.org/10.1002/ dvdy.20681

Geng JF, Liu X, Zhao HB, Fan WF, Geng JJ, Liu XZ (2018) LncRNA UCA1 inhibits epilepsy and seizure-induced brain injury by regulating miR-495/Nrf2-ARE signal pathway. Int J Biochem Cell Biol 99: 133-139. https://doi.org/10.1016/j.biocel.2018.03.021

Han CL, Ge M, Liu YP, Zhao XM, Wang KL, Chen N, Meng WJ, $\mathrm{Hu}$ W, Zhang JG, Li L, Meng FG (2018) LncRNA H19 contributes to hippocampal glial cell activation via JAK/STAT signaling in a rat model of temporal lobe epilepsy. J Neuroinflamm 15: 103-103. https://doi.org/10.1186/s12974-018-1139-z

Hébert SS, Horré K, Nicolaï L, Papadopoulou AS, Mandemakers W, Silahtaroglu AN, Kauppinen S, Delacourte A, De Strooper B (2008) Loss of microRNA cluster miR-29a/b-1 in sporadic Alzheimer's disease correlates with increased BACE1/beta-secretase expression. Proc Natl Acad Sci U S A. 105: 6415-6420. https://doi.org/10.1073/ pnas.0710263105

Henshall DC (2013) MicroRNAs in the pathophysiology and treatment of status epilepticus. Frontiers Mol Neurosic 6: 37. https://doi. org $/ 10.3389 /$ fnmol.2013.00037

Hodges SL, Lugo JN (2018) Wnt/ $\beta$-catenin signaling as a potential target for novel epilepsy therapies. Epilepsy Res 146: 9-16. https://doi. org/10.1016/j.eplepsyres.2018.07.002

Hu K, Xie YY, Zhang C, Ouyang DS, Long HY, Sun DN, Long LL, Feng L, Li Y, Xiao B (2012) MicroRNA expression profile of the hippocampus in a rat model of temporal lobe epilepsy and miR-34a-targeted neuroprotection against hippocampal neurone cell apoptosis post-status epilepticus. BMC Neurosi 13: 115. https://doi. org/10.1186/1471-2202-13-115

Jimenez-Mateos EM, Bray I, Sanz-Rodriguez A, Engel T, McKiernan RC, Mouri G, Tanaka K, Sano T, Saugstad JA, Simon RP, Stallings RL, Henshall DC (2011) miRNA Expression profile after status epilepticus and hippocampal neuroprotection by targeting miR-132. Am J Pathol 179: 2519-2532. https://doi.org/10.1016/j. ajpath.2011.07.036

Johnson R, Zuccato C, Belyaev ND, Guest DJ, Cattaneo E, Buckley NJ (2008) A microRNA-based gene dysregulation pathway in Huntington's disease. Neurobiol Dis. 29: 438-445. https://doi. org/10.1016/j.nbd.2007.11.001

Jones-Gotman M, Zatorre RJ, Olivier A, Andermann F, Cendes F, Staunton H, McMackin D, Siegel AM, Wieser HG (1997) Learning and retention of words and designs following excision from medial or lateral temporal-lobe structures. Neuropsychologia 35: 963-973. https://doi.org/10.1016/s0028-3932(97)00024-9

Kang W, Huang T, Zhou Y, Zhang J, Lung RWM, Tong JHM, Chan AWH, Zhang B, Wong CC, Wu F, Dong Y, Wang S, Yang W, Pan Y, Chak WP, Cheung AHK, Pang JCS, Yu J, Cheng ASL, To KF (2018) miR-375 is involved in Hippo pathway by targeting YAP1/ TEAD4-CTGF axis in gastric carcinogenesis. Cell Death Dis 9: 9292. https://doi.org/10.1038/s41419-017-0134-0

Karnati HK, Panigrahi MK, Gutti RK, Greig NH, Tamargo IA (2015) miRNAs: Key Players in Neurodegenerative Disorders and Epilepsy. J Alzheimer's Dis: JAD 48: 563-580. https://doi.org/10.3233/ JAD-150395

Kawano Y, Kypta R (2003) Secreted antagonists of the Wnt signalling pathway. J Cell Sci 116: 2627-2634. https://doi.org/10.1242/ jes.00623

Kele J, Andersson ER, Villaescusa JC, Cajanek L, Parish CL, Bonilla S, Toledo EM, Bryja V, Rubin JS, Shimono A, Arenas E (2012) SFRP1 and SFRP2 dose-dependently regulate midbrain dopamine neuron development in vivo and in embryonic stem cells. Stem Cells 30: 865-875. https://doi.org/10.1002/stem.1049

Lee DY, Moon J, Lee ST, Jung KH, Park DK, Yoo JS, Sunwoo JS, Byun JI, Lim JA, Kim TJ, Jung KY, Kim M, Jeon D, Chu K, Lee 
S (2015) Dysregulation of long non-coding RNAs in mouse models of localization-related epilepsy. Biochem Biophys Res Commun 462 433-440. https://doi.org/10.1016/j.bbrc.2015.04.149

Liu Y, Zhou D, Li G, Ming X, Tu Yf, Tian J, Lu H, Yu B (2015) Long non coding RNA-UCA1 contributes to cardiomyocyte apoptosis by suppression of p27 expression. Cell Physiol Biochem 35: 1986-1998. https://doi.org/10.1159/000374006

Ou J, Kou L, Liang L, Tang C (2017) MiR-375 attenuates injury of cerebral ischemia/reperfusion via targetting Ctgf. Biosci Rep 37: https://doi.org/10.1042/bsr20171242

Perkins KL, Arranz AM, Yamaguchi Y, Hrabetova S (2017) Brain extracellular space, hyaluronan, and the prevention of epileptic seizures. Rev Neurosci 28: 869-892. https://doi.org/10.1515/revneuro-2017-0017

Qiao S, Xu Q, Cheng L, Zhang Y, Liu X, Zheng C, Hu X, Chen $\mathrm{L}$ (2018) Integrated analysis of differentially expressed lncRNAs in Medial Temporal Lobe Epilepsy. Neuro Endocrinol Lett 39: 119-124. PMID: 30183206

Rubio C, Taddei E, Acosta J, Custodio V, Paz C (2020) Neuronal excitability in epileptogenic zones regulated by the Wnt/B-catenin pathway. CNS Neurol Disord Drug Targets 19: 2-11. https://doi.org/1 $0.2174 / 1871527319666200120143133$

Tai XY, Bernhardt B, Thom M, Thompson P, Baxendale S, Koepp M, Bernasconi N (2018) Review: Neurodegenerative processes in temporal lobe epilepsy with hippocampal sclerosis: Clinical, pathological and neuroimaging evidence. Neuropathol Appl Neurobiol 44: 70-90. https://doi.org/10.1111/nan.12458

Villa C, Lavitrano M, Combi R (2019) Long non-coding RNAs and related molecular pathways in the pathogenesis of epilepsy. Int J Mol Sci 20: 4898. https://doi.org/10.3390/ijms20194898

Wang H, Yao G, Li L, Ma Z, Chen J, Chen W (2020) LncRNA-UCA1 inhibits the astrocyte activation in the temporal lobe epilepsy via regulating the JAK/STAT signaling pathway. I Cell Biochem 121: 4261-4270 https://doi.org/10.1002/jcb.29634

Wang Y, Lieberman R, Pan J, Zhang Q, Du M, Zhang P, Nevalainen M, Kohli M, Shenoy NK, Meng H, You M, Wang L (2016) miR375 induces docetaxel resistance in prostate cancer by targeting SEC23A and YAP1. Mol Cancer 15: 70-70. https://doi.org/10.1186/ s12943-016-0556-9

Wu X, Wang Y, Sun Z, Ren S, Yang W, Deng Y, Tian C, Yu Y, Gao (2019) Molecular expression and functional analysis of genes in children with temporal lobe epilepsy. J Integr Neurosici 18: 71-77. https://doi.org/10.31083/j.jin.2019.01.13

Yu Q, Zhao MW, Yang P (2020) LncRNA UCA1 suppresses the inflammation via modulating miR-203-mediated regulation of MEF2C/NF- $x$ B signaling pathway in epilepsy. 45: 783-795. https://doi.org/10.1007/s11064-019-02952-9

Zheng J, Yi D, Liu Y, Wang M, Zhu Y, Shi H (2017) Long nonding RNA UCA1 regulates neural stem cell differentiation by controlling miR-1/Hes1 expression. Am J Transl Res 9: 3696-3704. PMID: 28861160 